\title{
Estudio comparativo entre el índice de masa corporal y el control postural en alumnos sanos de la carrera de Terapia Física de la Universidad Politécnica de Pachuca
}

\section{Comparative study between body mass index and postural control in healthy students of the Physical Therapy degree at the Universidad Politécnica de Pachuca}

\author{
VÁZQUEZ-CHACÓN, Verónica †**, TERRAZAS-LÓPEZ, Guillermo y SÁNCHEZ-BARRERA, \\ Erendira
}

\section{Universidad Politécnica de Pachuca}

ID $1^{\text {er }}$ Autor: Verónica, Vázquez-Chacón / ORC ID: 0000-0003-2002-6876, Researcher ID Thomson: V-5652-2018

ID $1^{\text {er }}$ Coautor: Guillermo, Terrazas-López / ORC ID: 0000-0002-0149-2428

ID $2^{\text {do }}$ Coautor: Erendira, Sánchez-Barrera / ORC ID: 0000-0003-2466-6977

DOI: $10.35429 /$ JP.2019.10.3.27.33

Recibido 26 de Octubre, 2019; Aceptado 30 de Diciembre, 2019

\begin{abstract}
Resumen
Objetivo: Analizar en una población de jóvenes universitarios de la Licenciatura en Terapia Física de la Universidad Politécnica de Pachuca, la influencia del IMC en la estabilidad postural, antero-posterior, mediolateral y general por medio de la utilización del sistema Balance SD de Biodex. Método: Se realizaron medias de talla, peso IMC de todos los individuos, se presentaron datos por medio de tablas de registro, posteriormente se clasificaron los resultados de acuerdo al IMC, separándolos en grupos de IMC bajo, normal y sobrepeso, y se compararon sus resultados de la prueba de estabilidad postural entre estos grupos normal y se presentaron los resultados gráficamente. Resultados: De acuerdo a las comparaciones se pudo notar que el sobrepeso es determinante en el control postural, principalmente en el Índice antero-posterior con un puntaje mayor .06 sobre los de IMC normal y de .1 de los de IMC bajo.
\end{abstract}

IMC, Equilibrio, Biodex

\begin{abstract}
Objective: To analyze in a population of young university students of the Degree in Physical Therapy of the Polytechnic University of Pachuca the influence of BMI on postural, antero-posterior, mid-lateral and general stability through the use of Biodex Balance SD system. Method: Measurements of height BMI weight of all individuals were performed, data were presented by means of registration tables, then the results were classified according to the BMI that presented each of them separating them into low, normal and overweight BMI groups and their postural stability test results were compared between these normal groups and the results presented graphically. Results: According to the comparisons, it was noted that overweight is a determining factor in postural control, mainly in the anteroposterior index with a score greater than .06 over those of normal BMI and .1 of those of low.
\end{abstract}

Balance body mass index, Biodex

Citación: VÁZQUEZ-CHACÓN, Verónica, TERRAZAS-LÓPEZ, Guillermo y SÁNCHEZ-BARRERA, Erendira. Estudio comparativo entre el índice de masa corporal y el control postural en alumnos sanos de la carrera de Terapia Física de la Universidad Politécnica de Pachuca. Revista de Fisioterapia. 2019. 3-10: 27-33

\footnotetext{
* Correspondence to Author (email: veronicavazquez@upp.edu.mx)

$\dagger$ Researcher contributing first author.
} 


\section{Introducción}

El equilibrio, es una parte esencial del ser humano, tanto como para realizar todo tipo de actividades de la vida diaria. Algunos grupos de personas por su profesión, están obligados a tener un buen equilibrio y un buen control de la postura, los estudiantes de Terapia Física entran en este seleccionado grupo de personas, sobre todo, para ser capaces de realizar y ejemplificar cada uno de los ejercicios que pueden ser indicados al paciente, es por eso que, además de un buen control postural los estudiantes de terapia física deben tener una buena condición física y por ende un buen puntaje en el Índice de masa corporal (IMC).

Este artículo, tiene su fundamentación teórica en seis categorías conceptuales: Obesidad, Índice de Masa Corporal, Equilibrio, Postura, Terapia Física y sistema Balance SD de Biodex, seguido de la fundamentación teórica, se describen los pormenores de la investigación, incluyendo las comparaciones de los índices de obesidad e índices de estabilidad.

Dentro de la presente investigación, se analizarán los factores (antecedentes personales de riesgo para alteraciones de equilibrio); La presente investigación, está encaminada a crear conciencia, tanto en alumnos, como docentes de la Licenciatura en Terapia Física para mantenimiento de control de peso, para influencia positiva en el ámbito laboral.

En antecedentes investigativos, únicamente se ha llevado a cabo una investigación en España, por Gallego Gómez et al., en el 2011, quisimos replicar para obtener información en México.

\section{Desarrollo}

La obesidad, se entiende como la condición en que una persona tiene una cantidad excesiva de grasa corporal y es perjudicial para la salud, es como consecuencia de un mayor aporte de energía en relación a su consumo, en México se ha declarado una emergencia sanitaria por la epidemia de obesidad y diabetes. En 2016, $72.5 \%$ de los adultos presentaron sobrepeso y obesidad, y aun cuando desde 1999 se ha observado un incremento en toda la población.
La obesidad aumenta el riesgo de padecer otras enfermedades como: diabetes mellitus, enfermedad isquémica del corazón, hipertensión, dislipidemias, enfermedades cerebrovasculares y cáncer, las cuales disminuyen la calidad de vida e incrementan el riesgo de muerte prematura entre quienes las padecen. La obesidad también representa altos costos médicos, estimados en 151894 millones de pesos sólo en 2014, lo cual, equivale a $34 \%$ del gasto público en salud; El objetivo del tratamiento de esta enfermedad es la reducción de peso en adultos con obesidad y orientados a la pérdida y mantenimiento del peso a largo plazo, este peso no debe ser mayor a $1 \mathrm{~kg}$ por semana generalmente.

El IMC, es un indicador conveniente y confiable para estimar la obesidad de una persona, y se determina dividiendo el peso corporal en kilogramos por el cuadrado de la estatura corporal en metros.

$\mathrm{IMC}=$ Peso en Kilogramos $/($ Talla en $\mathrm{cm}) 2$

\begin{tabular}{|l|r|r|}
\hline \multicolumn{1}{|c|}{ Clasificación } & \multicolumn{1}{|c|}{ IMC } & \multicolumn{2}{c|}{$\begin{array}{c}\text { Tipo de } \\
\text { Obesidad }\end{array}$} \\
\hline Delgadez & $<18,5$ & \\
\hline Peso normal & $18,5-24,9$ & \\
\hline Sobrepeso & $25,0-29,9$ & I \\
\hline Obesidad & $30,0-34,9$ & II \\
\hline \begin{tabular}{l|r} 
Obesidad \\
extrema
\end{tabular} & $35,0-39,9$ & III \\
\hline
\end{tabular}

Tabla 1 Clasificación de sobrepeso y obesidad por IMC Fuente: Jack $h$ wilmore, J.H.W, David $l$ ostill, D.L.O. Fisiología del esfuerzo y del deporte. (6ta edición ed.). Baladona España: Paidotribo; 2010, p. 714

El Equilibrio, es la capacidad del hombre de mantener su propio cuerpo, otro cuerpo (u objetos) en una posición controlada y estable, por medio de movimientos compensatorios, distinguiéndose entre el equilibrio estático, dinámico y la capacidad de mantener en equilibrio un cuerpo extraño $u$ objeto; este mantiene el centro de gravedad dentro de la base de apoyo del cuerpo y requiere ajustes constantes que son proporcionados por la actividad muscular, y la posición de las articulaciones. La seguridad o precariedad de este estado de equilibrio depende sobre todo de la relación entre el centro de la gravedad del objeto y su base de sustentación. 
Existen 3 estados del equilibrio, estable, inestable y neutro: el equilibrio estable cuando un objeto está en una posición en que, para alterarla, hay que elevar su centro de gravedad, el equilibrio inestable cuando se necesita una fuerza mínima para trastocar el objeto y el neutro cuando el centro de gravedad no se eleva ni desciende cuando se altera.

La postura se define como la posición relativa de las diferentes partes del cuerpo con respecto a sí mismas (el sistema coordinado egocéntrico), al ambiente (el sistema coordinado exocéntrico) o al campo gravitatorio (el sistema coordinado geocéntrico). La postura corporal es inherente al ser humano y una buena postura conlleva que las partes corporales están en las posiciones que más favorecen la función, esas posiciones equilibran la distribución del peso y por tanto imponen menos carga a los músculos, tendones, ligamentos y huesos.

El control de la postura ortostática (postura estática, bípeda) es la base para la ejecución de infinidad de tareas motoras utilizadas en la vida diaria o durante la práctica deportiva. Este mecanismo representa la habilidad del sujeto para mantener la posición del cuerpo y específicamente su centro de masas dentro de los límites de seguridad respecto a lo que representa la posición erecta del cuerpo.

La Fisioterapia es definida, como el arte y la ciencia del tratamiento por medio del ejercicio terapéutico, calor, frio, agua, masaje, electricidad, el alivio del dolor, aumento de la circulación, prevención y corrección de la disfunción, máxima recuperación de la fuerza, movilidad y coordinación. El Licenciado en Terapia Física colabora con el equipo interdisciplinario de salud en la elaboración de los planes de tratamiento rehabilitatorio integral, tanto del individuo discapacitado como con el sujeto de riesgo, con la aplicación de técnicas generales y específicas de rehabilitación.

\section{Metodología}

Es una investigación de tipo transversal, cuantitativa, descriptiva, con muestreo no probabilístico estratificado por conveniencia.

\section{Material}

Para la medición de las características antropométricas, se utilizó una báscula inteligente marca Huawei versión AH100 y una báscula con tallímetro para adultos.

Para la realización del test de estabilidad postural se utilizó la plataforma estabilométrica del sistema Balance SD de Biodex. El parámetro 0 es indicativo de un buen control postural y conforme se alejan los valores de 0 es indicativo de una peor estabilidad postural.

\section{Método}

El cálculo del IMC se realizó en una hoja de Excel.

Los datos obtenidos en la muestra, se obtuvieron con los sujetos con ropa ligera, sin zapatos ni objetos que pudieran alterar los resultados como celulares monederos $\mathrm{o}$ carteras; posteriormente, se obtuvieron los datos arrojados por el sistema Balance SD de Biodex, instruimos a los sujetos para manutención de su centro de gravedad en un punto fijo establecido por una pantalla situada a $30 \mathrm{~cm}$ delante de su cara.

Se realizaron 3 comparaciones, derivado de la división en 3 grupos de la muestra, como se muestra a continuación:

Grupo 1: $100 \%$ de la muestra, incluye personas con IMC bajo, IMC normal e IMC en sobre peso.

Grupo 2: Del $100 \%$ personas se eliminaron a las que presentaron antecedentes patológicos que pudieran alterar el equilibrio, se dividió en IMC bajo, normal y sobre peso.

Grupo 3: Personas con antecedentes patológicos $\mathrm{y}$ personas sin antecedentes patológicos.

\section{Descripción de la muestra}

Los sujetos participantes en este estudio fueron 57 alumnos, con un promedio de IMC de 25.3466, de ellos, $84.21 \%$ mujeres, con un IMC de 25.0923 y $15.79 \%$ hombres, con un IMC de 26.7027, con un mínimo de edad de 20 años y un máximo de edad de 32 años de edad, promedio de edad de 21.38 . 
A cada participante se le realizó una breve historia clínica y se le dio a firmar un consentimiento informado.

\section{Resultados}

Se obtuvo el peso y la talla de cada individuo y mediante la fórmula "IMC $=$ Peso en Kilogramos / (Talla en cm)2" se obtuvo el IMC de cada individuo y fueron separados los resultados de acuerdo a la clasificación de la OMS para el IMC, con una media de 25.34 $\mathrm{kg} / \mathrm{m} 2$, el cual, corresponde a una situación de sobrepeso. Las variables relacionadas con la estabilidad postural están determinadas por el plano frontal, para el índice medio-lateral, por el plano sagital para el índice antero-posterior y la suma de estos dos parámetros dan como resultado el índice de estabilidad general de cada individuo.

Se obtuvieron 3 resultados: El primero, el índice de estabilidad general (IEG) determinado por los resultados de índice medio lateral (IML) e índice antero posterior (IAP), el segundo que se obtuvo es el \% en zona (A, B, C o D), el cual indica el \% de tiempo en el que el individuo se mantuvo en determinada zona y el tercero y último es el \% de zona en cuadrante (I, II, III, IV) en el que el individuo estuvo durante las pruebas.

\section{Análisis de resultados por grupos}

\section{Grupo 1}

\section{Índice de estabilidad}

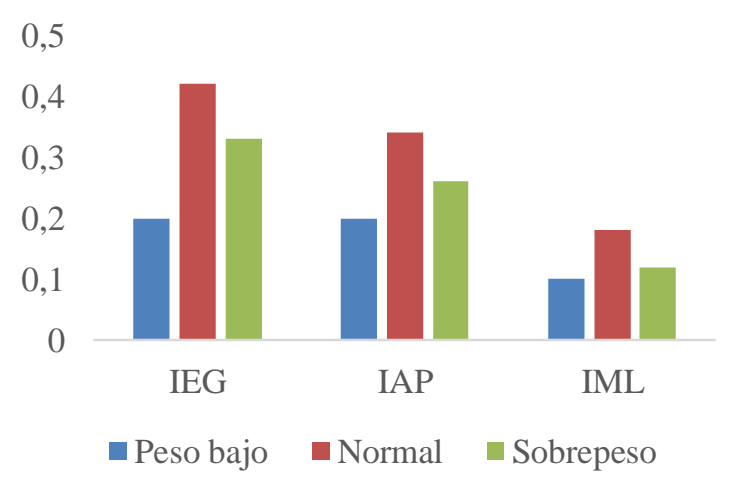

Gráfico 1 Promedio de índices de estabilidad Grupo 1 Fuente: Autoría Propia

Las personas con IMC normal tienen peor control postural con un puntaje de diferencia de $.09 \%$ que los de sobrepeso, .22\% de diferencia, con respecto a los de peso bajo, siendo estos, los de mejor control postural.
De los individuos de peso normal, 92\%, obtuvieron un $100 \%$ de tiempo en la zona A, y solo un $8 \%$ presentó distinto resultado, y sus resultados fueron 1: zona: A 93\%, B 7\%, C 0\%, D 0\%; 2: A98\%, B1\%, C1, D0\%. Por otra parte, De los individuos con sobrepeso el $96 \%$ obtuvieron un $100 \%$ en la zona A y sólo el $4 \%$ con diferente resultado como sigue: A 99\%, B $1 \%, \mathrm{C} 0 \%$, D $0 \%$.

\section{Porcentaje de tiempo en cuadrante}

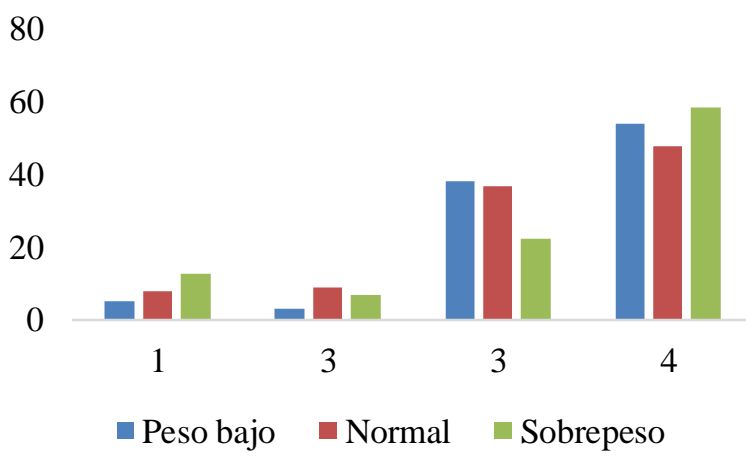

Gráfico 2 Porcentaje de tiempo en cuadrante Grupo 1 Fuente: Autoría propia

Las personas con sobrepeso obtuvieron un mayor porcentaje de tiempo en los cuadrantes 1 y 4, correspondientes al eje anteroposterior del lado derecho.

\section{Grupo 2}

A continuación, se enuncian los resultados de índices de estabilidad obtenidos en el grupo 2, sin antecedentes que afecten el equilibrio, como sigue:

\section{Índice de estabilidad}

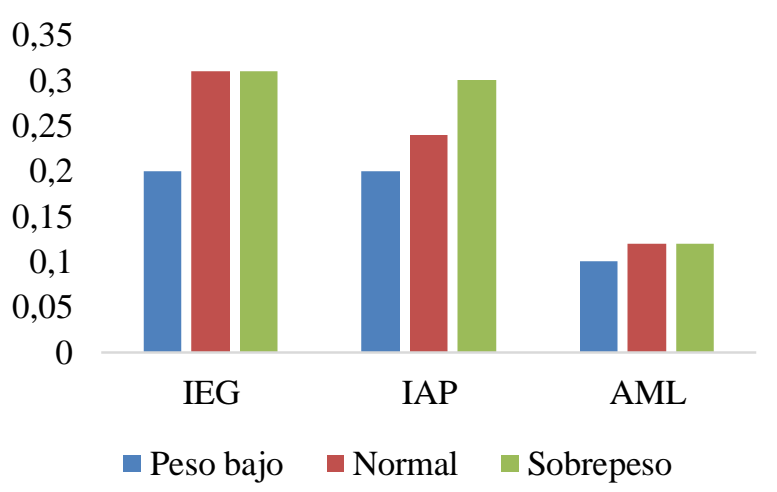

Gráfico 3 Promedio de Índices de estabilidad del Grupo 2

Fuente: Autoría Propia 
Los sujetos de peso bajo, presentan mejor control postural, mientas que los de sobrepeso, presentan un puntaje más alto en el índice de estabilidad antero-posterior, con una puntuación de diferencia de $.06 \%$, siendo indicativo de que las personas con sobrepeso tienen peor control postural.

\section{Porcentaje de tiempo en cuadrante}

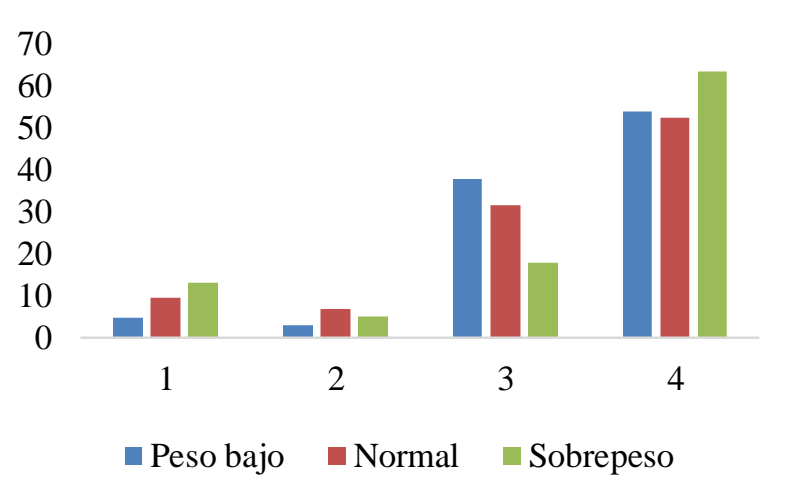

Gráfica 4 Porcentaje de tiempo en cuadrante Grupo 2 Fuente: Autoría Propia

Se puede notar que las personas con sobre peso presentan mayor porcentaje en el cuadrante 1 y 4 correspondiente al eje anteroposterior del lado derecho, mientras que en el cuadrante 2 predominan los de peso normal en el 3 los de peso bajo. En cuanto al porcentaje de tiempo en zona no se encontraron datos significativos.

\section{Grupo 3}

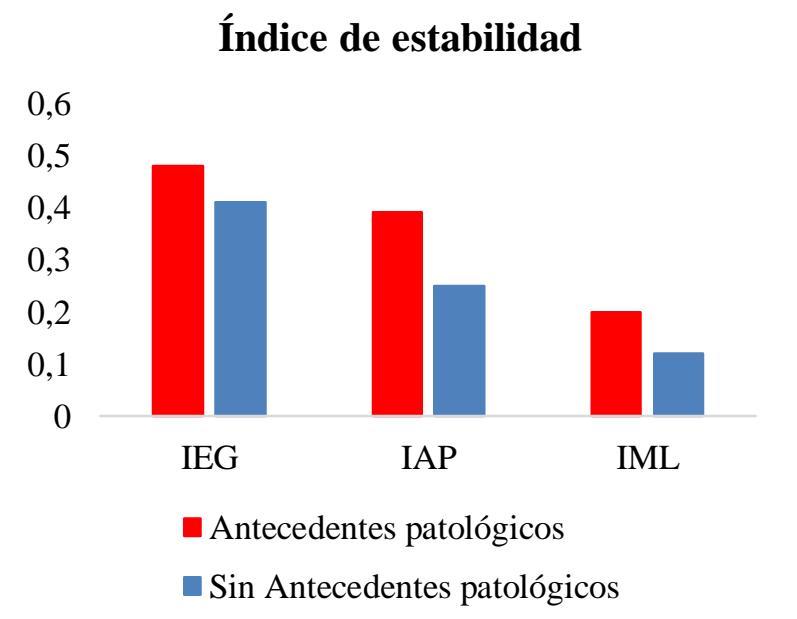

Gráfica 5 Promedio de Índices de estabilidad del Grupo 3

Fuente: Autoría Propia
Los individuos con antecedentes patológicos tuvieron un mayor puntaje en todos los índices con respecto a los que no, teniendo una diferencia mayor de $.08 \%$ en el general, de $.14 \%$ en el antero-posterior y de .08 en el medio-lateral, convirtiéndose en los individuos con peor control postural de este estudio.

En cuanto al porcentaje de tiempo en zona el $100 \%$ de las personas sin antecedente, obtuvieron un $100 \%$ en la zona A y del $100 \%$ de individuos que presentaron antecedentes patológicos que pueden alterar el equilibrio, el $85 \%$ tuvieron un $100 \%$ en la zona A mientras que los otros $15 \%$, sus resultados fueron: 1: A $93 \%$, B 7\%, C 0\%, D 0\%; 2: A 98\%, B 1\%, C1\%, D 0\%; 3: A 99\%, B 1\%, C 0\%, D 0\%.

\section{Porcentaje de tiempo en cuadrante}

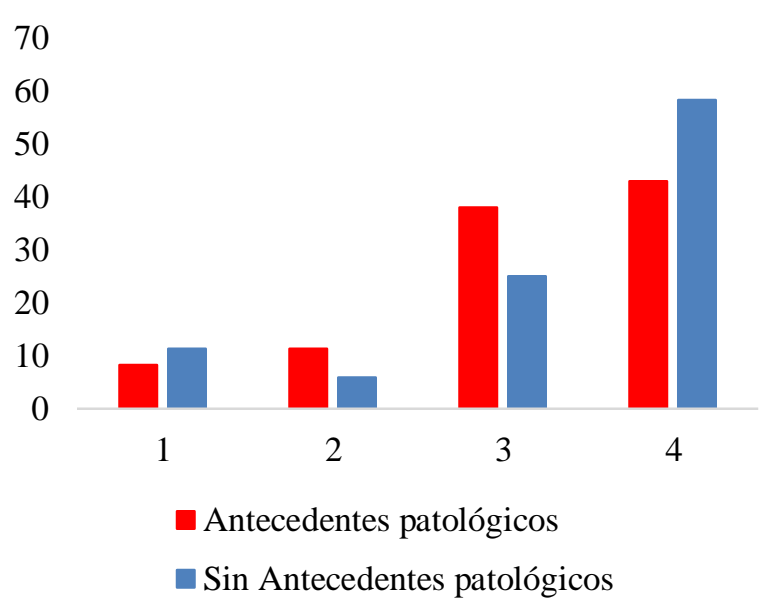

Gráfico 6 Porcentaje de tiempo en cuadrante Grupo 3 Fuente: Autoría propia

Las personas sin antecedentes predominan en los cuadrantes 1 y 4 , en el cuadrante 2 y 3 predominan los que tienen antecedentes personales patológicos, lo que indica que los que tienen antecedentes tienen una carga de peso a la izquierda mientras que los que no presentan antecedentes presentan la carga de peso a la derecha.

\section{Agradecimientos}

Los autores agradecen a la Mtra. Rosa María Chío Austria, Directora del Programa Educativo de la Licenciatura en Terapia Física de la Universidad Politécnica de Pachuca, por las facilidades para la realización de la presente investigación. 


\section{Referencias}

A Zugasti Murillo, Eduardo David Creus García. (enero 2005). Obesidad como factor de riesgo cardiovascular. ELSEVIER, 1, 36. 13 de agosto del 2019, De ELSEVIER Base de datos.

Ana Julia García Milian, Eduardo David Creus García. (7 de marzo del 2016). La obesidad como factor de riesgo, sus determinantes y tratamiento. Revista Cubana de Medicina General Integral, 3, 13. 12 de agosto del 2019, De ELSEVIER Base de datos.

Arthur c guyton . (1997). Anatomía y fisiología del sistema nervioso. España: Médica Panamericana.

Barney Le Veau. (2016). B.L.V Biomecanica del movimiento humano. (1 ${ }^{\mathrm{a}}$ ed.).. Mexico, DF: Trillas.

Carlos oropeza abúndez. (2018). La obesidad en México Estado de la política pública y recomendaciones para su prevención y control. Cuernavaca, Morelos, México: Instituto Nacional de Salud Pública.

Catherine Parker, Gary A. Thibode,. (1883). Anatomía y Fisiología. San francisco, CA: MC Graw Hill.

Eduardo loría, Emmanuel salas. (2014). Sobrepeso e integración económica en México. Economía Informa, 389, 16. 2014, De Economía Informa Base de datos.

Elaine n marieb. (2006). Essentials of human anatomy \& physiology. San francisco, CA : Pearson.

Elaine n marieb. (2008). Anatomía y fisiología humana. Madrid España: Pearson.

Florence Peaterson Kendall, Elizabeth Kendall McCreary, Patricia Geise Provance, Williams Anthony Romani. (2007). Músculos Pruebas Funcionales Postura y Dolor. México, D.F: MARBÁN.

Gobierno federal . (2015). Estrategia parala Nutrición, Actividad Fisica y Prevencion de la Obesidad (NAOS). 11 agosto 2019, de Agencia española de Seguridad Alimentaria y Nutricion (AESAN) Sitio web: http://www.naos.aesan. msssi.gob.es/naos/estrategia/que.es/
Hall e john, David 1 ostill. (2016). Tratado de fisiología médica. Barcelona España: ELSEVIER.

Hue O, Simoneau M, Marcotte J, Berrigan F, Doré J, Marceau P, et al.. (2007). Body weight is a strong predictor of postural stability. PubMed, 32-8, 1. 22 agosto 2006, De Gaut Posture Base de datos.

Jack h wilmore, David 1 ostill. (2010). Fisiología del esfuerzo y del deporte. Baladona España: Paidotribo.

Kevin T Patton, Gary Thibodeau. (1988). Estructura y Función del cuerpo Humano. Barcelona España: ELSEVIER.

Margarita torres tamayo. (3 de octubre de 2004). ¿Cuáles son los factores de riesgo a que conlleva la obesidad? Revista de Endocrinología y Nutrición, 12, 4. 13 de agosto del 2019, De mediagraphic.com Base de datos.

Michael Kent. (2003). Diccionario Oxxford de medicina y ciencias del deporte. ( $1^{\text {a }}$ edición). Barcelona España: Paidotribo.

Mónica Martínez Olguín (2012.) Discrepancia entre el perfil de egreso universitario del terapeuta físico y sus competencias laborales en instituciones publicas de salud en México [tesis] Zempoala, Hidalgo: Universidad Plotecnica de Pachuca.

Rodrigo Cano, Soriano del Castillo. (8 de junio de 2017). Causas y tratamiento de la obesidad. Nutrición clínica y dietética hospitalaria, 4, 6. 11 de agosto del 2019, De ELSEVIER Base de datos.

Rubio M. A., Salas-Salvadó J, ArancetaJ. (marzo 2007). Consenso SEEDO 2007 para la evaluación del sobrepeso y la obesidad y el establecimiento de criterios de intervención terapéutica. Revista Española de Obesidad, 1, 52. 11 de agosto del 2019, De SEEDO Base de datos.

SalvadorJetal. (2012). Prevención, diagnóstico y tratamiento de la obesidad. Gobierno Federal, 2, 82. 11 de agosto del 2019, De Secretaria de Salud Base de datos. 
ShuklaAP, BuniakWI, AronneLJ. (2015) Treatment of obesity in 2015, 35,92. $2015 \mathrm{~J}$ Cardiopulm Rehabil Prev.

Whoint. (2019). Licenciatura en Terapia Física. 18 de agosto de 2019, de Universidad Politécnica de Pachuca Sitio web: http://www.upp.edu.mx/ofertaeducativa/?page $\mathrm{id}=24$

Whoint. (2019). Obesidad y sobrepeso. 17 agosto 2019, de Organización Mundial de la Salud Sitio web: https://www.who.int/es/newsroom/fact-sheets/detail/obesity-and-overweight. Whoint. Whoint [Online]. (2018). Malnutricion. 16 febrero 2018, de Organización Mundial de Salud Sitio web: https://www.who.int/es/newsroom/fact-sheets/detail/malnutrition 